\title{
A Case Report of Extensively Drug Resistant Typhoid in Karachi, Pakistan: A Major Health Concern to Curb the Outbreak
}

\author{
Authors: \\ *Sarah Kamran Akbani, Fazeela Bibi \\ Jinnah Medical and Dental College, Karachi, Pakistan \\ *Correspondence to sarah.akbani@hotmail.com \\ Disclosure: \\ The authors have declared no conflicts of interest. \\ Received: \\ 30.03 .21 \\ Accepted: \\ 20.07.21 \\ Keywords: \\ Child, developing countries, drinking water, meropenem, Salmonella typhi, typhoid \\ fever. \\ Citation: \\ EMJ 2021;6[3]:125-129.
}

\begin{abstract}
The disease burden of extensively drug resistant typhoid in developing countries is a major emerging issue that cannot be ignored. Since its emergence from multidrug strains, the majority of typhoid cases in Karachi, Pakistan, have been extensively drug resistant, mostly infecting younger patients. In the study, the authors analysed one such case in an adolescent male and discussed how, by the implementation of national health policies, the spread of these infectious diseases could be prevented and the overall burden on the healthcare system decreased in areas with already limited resources.
\end{abstract}

\section{INTRODUCTION}

Salmonella typhi is a flagellated gram-negative bacterium that is rod shaped and causes typhoid fever in its only host: humans. ${ }^{1}$ The transmission of typhoid fever is mainly through "direct, person to person transmission as a result of improper hygiene and unsafe food/water handling practices" causing faeco-oral spread of the bacterium especially in "densely populated areas that lack proper sanitation and access to safe drinking water" such as in Pakistan. ${ }^{2}$ After the ingestion of $S$. typhi, the bacterium enters the systemic circulation by crossing through the epithelium of the intestine and can disseminate to the liver, gall bladder, bone marrow, and spleen, causing symptoms such as fever that may become life threatening, generalised muscle ache, abdominal pain, and headache. ${ }^{3}$

The treatment of typhoid is primarily through antibiotics; however, drug resistance to common antibiotics is increasing rapidly, causing major concern. ${ }^{4}$

Extensively drug resistant (XDR) typhoid fever has become a major health issue in some parts of the world since it began in late 2016 in Hyderabad district of Sindh, Pakistan, due to strains of $S$. typhi that are antimicrobial resistant $(A M R))^{5}$ The emergence of XDR typhoid from the already concerning multidrug resistant typhoid has occurred due to the organism simply obtaining a plasmid that is now resistant to multiple classes of antibiotics including first-line and second-line treatment options such as trimethoprim-sulfamethoxazole, ampicillin, chloramphenicol, third generation cephalosporins, and fluoroquinolones. ${ }^{6-8}$ it is 
hypothesised that the spread of this XDR type occurred because the $S$. typhi strain acquired this plasmid from Escherichia coli, since researchers discovered that the isolated plasmid resembled one within a Nigerian E. coli sample. ${ }^{7}$

In Karachi, Pakistan, the incidence of XDR typhoid cases reported in major hospitals since the initial outbreak was 7,622 of out of a total 11,717 cases of typhoid, according to the National Institute of Health $(\mathrm{NIH})$ Islamabad. ${ }^{9}$ Out of these, most $(42 \%)$ of those affected were children under the age of 4 years. ${ }^{9}$ The overall attack rate was $38 / 100,000$ population, the highest of which was found in 0-4 years age, group with 116/100,000 population, followed by the 5-9 years age group with $83 / 100,000$ population. ${ }^{9}$

\section{CASE REPORT}

A previously healthy 13-year-old male, a resident of Korangi, Karachi, Pakistan, presented in the outpatient department on $13^{\text {th }}$ August 2019, with the complaint of sudden onset high grade, stepladder fever for 2 weeks. It was initially 101$102{ }^{\circ} \mathrm{F}$ later climbing to $103^{\circ} \mathrm{F}$ over the span of 8 days with associated rigors, chills, abdominal pain, cough, congestion, and generalised body ache. On admission, his vitals included blood pressure: 114/68 mmHg; temperature: $104{ }^{\circ} \mathrm{F}$; pulse: 62 beats per minute; respiratory rate: 14 breaths per minute; oxygen saturation on room air: $97 \%$; and his weight was $60 \mathrm{~kg}$. The patient was alert and oriented with time, place, and person, and his Glasgow Coma Scale (GCS) was 15/15. Physical examination was remarkable for pallor, mild muscle tenderness, and abdominal tenderness, and negative for cyanosis, clubbing, oedema, icterus, lymphadenopathy, splenomegaly, and skin rash. Upon systemic examination, the cardiovascular and respiratory systems had no significant findings. His abdomen was tender with guarding present due to pain, and there was no visceromegaly. There were no signs of ascites, and normal bowel sounds were present. His neurological examination was normal. He had no bone or joint pains or swelling.

The child was prescribed paracetamol per os, si opus sit, and advised for investigation including complete blood count and posterior anterior chest X-ray chest PA. On the third day, when symptoms did not subside, the family came for a follow-up. The child was admitted and other investigations including a blood culture, dengue non-structural protein 1 antigen, malarial parasite, urine detailed report, urea, creatinine, electrolytes, liver function tests, and viral marker were sent. The child's vitals were monitored, and he was started on loratadine $10 \mathrm{mg}$ per os once daily, nebulised ipratropium bromide every 6 hour for cough and congestion, and IV Ringer's lactate $75 \mathrm{~mL} /$ hour, IV paracetamol $1 \mathrm{~g}$ si opus sit, IV ceftriaxone $1 \mathrm{~g}$ twice daily for fever and antibiotic cover. Investigations revealed only mild anaemia and platelet count of $131 \times 10^{9} / \mathrm{L}$, as well as, a drop in leukocytes from $7.6 \times 10^{9} / \mathrm{L}$ to $3.8 \times 10^{9} / \mathrm{L}$. Aspartate transaminase, alanine transaminase, and $\mathrm{y}$-glutamyl transferase were deranged on liver function tests.

The chest X-ray was clear and urea, creatinine, electrolytes, and urine detailed report were normal, and hepatitis markers, dengue nonstructural protein 1 antigen and malarial parasite were negative. The blood culture revealed growth of S. typhi that was resistant to ampicillin, ciprofloxacin, cefotaxime, co-trimoxazole, cefixime, ceftriaxone, and chloramphenicol. This strain was only sensitive to azithromycin, imipenem, and meropenem. The patient was switched from ceftriaxone to IV imipenem/ cisplatin $500 \mathrm{mg}$ three times a day in 1,000 mL normal saline according to sensitivity with plan to de-escalate to tablet azithromycin $500 \mathrm{mg}$ twice daily. He was also given two capsules of loperamide stat, dioctahedral smectite powder twice daily, and miconazole oral gel three times a day for the complaints of diarrhoea and oral ulcers.

Fever subsided after 6 days of changing antibiotics. IV Imipenem/cisplatin was continued for a total of 7 days and then de-escalated to tablets of azithromycin when the patient's clinical condition was noted to be improving. Treatment was continued to complete a total of 14 days of therapy according to treatment guidelines set forth by the Medical Microbiology and Infectious Diseases Society of Pakistan. ${ }^{10}$ The patient's general condition improved; he was counselled on the importance of hygienic practices for the prevention of transmissible diseases and was discharged. 


\section{DISCUSSION}

Since the emergence of extensively drug resistant typhoid fever in Pakistan, the number of XDR cases has increasingly outnumbered sensitive cases, making it a progressively concerning issue in South Asia, specifically Karachi, Pakistan. The mechanism of AMR occurs via the plasmids in S. typhi $\mathrm{H} 58$ haplotype that carry resistant genes including trimethoprim-sulfamethoxazole resistance ( $d f r A 7$, sul1, sul2), ampicillin resistance (blaTEM-1), chloramphenicol resistance (catA7), fluoroquinolone resistance (qnr, oqxAB, or aac $\left.\left(6^{\prime}\right) \mid \mathrm{b}-\mathrm{cr}\right)$, and ceftriaxone resistance (ESBL)."1 These resistant genes have caused XDR typhoid to become a greater burden (about $72 \%$ more burdensome) than sensitive typhoid in Pakistan, specifically in terms of cost related to diagnosis and nursing care. ${ }^{12}$ In a study on the frequency of XDR typhoid fever, of total 969 typhoid fever cases, 777 (80.2\%) were multidrug resistantcases and showed resistance to all three firstline antibiotics (ampicillin, chloramphenicol, and co-trimoxazole); 517 (53.3\%) were XDR-typhoid fever cases, which were resistant to first- and second-line drugs (ciprofloxacin) and the third generation cephalosporin (ceftriaxone). Only two antibiotics (azithromycin and imipenem) were seen to be sensitive to all S. typhi cases. ${ }^{13}$ Many studies have suggested the use of azithromycin, carbapenems, and tigecycline for treatment of XDR typhoid.,14,15 This "sudden emergence and rapid spread of resistant isolates underline the importance of AMR surveillance for typhoid and other enteric Gram-negative bacteria and highlight the inadequacy of relying solely on non-culture-based methods for diagnosis of typhoid (such as Widal and Typhidot tests), which do not provide susceptibility results." Thus, guidelines to counteract the resistance have been set by the NIH Islamabad, which stress on the prompt confirmation of diagnosis of any and all suspected cases with blood culture and sensitivity prior to initiating empirical therapy. ${ }^{16}$ Furthermore, it is advised that the appropriate use of specific antibiotics should be based on the culture and sensitivity reports, and to refrain from using stronger antibiotics such as azithromycin or carbapenem for uncomplicated or sensitive cases. ${ }^{17}$

In the authors' case, they ensured proper investigations, including a blood culture, were conducted prior to beginning empiric therapy with the recommended antibiotic. After obtaining results of the blood culture and sensitivity, the authors promptly switched antibiotic management according to the appropriate sensitivities. It is the duty of healthcare professionals to fulfil a role in halting the spread of drug resistant organisms by following the guidelines set to diagnose and treat typhoid promptly and accurately.

The World Health Organization (WHO) has also highlighted some major modifiable risk factors to contain the spread of typhoid. These include the limited resources in rural set ups to screen and confirm the diagnosis of typhoid fever, the substandard prescription and use of antibiotics, unsanitary practices, and the inadequate use of vaccines among those at high risk, notably direct contacts with patients and carriers or those in high endemic incidence areas. ${ }^{2}$ These issues must be acknowledged and updated in Pakistan in order to curb the growing number of cases.

Poor sanitation is one of the major preventable factors contributing to the outbreak of typhoid, especially of drinking water when it is contaminated with sewage lines, increasing the risk of faeco-oral transmission of the bacteria. ${ }^{18}$ This is heightened by poverty, overcrowding, and a lack of education in proper hygiene. ${ }^{19}$ Some recommendations made by the $\mathrm{NIH}$ Islamabad include proper hand hygiene, using water that is filtered or boiled, and thoroughly washing produce prior to meal preparation. ${ }^{17}$ The importance of hygiene is exemplified by the nations, including Europe, North America, South America, and Australia, that have proper clean water and sewage line systems, and implement a national typhoid fever surveillance. ${ }^{20,21}$ Thus, sanitation and education about its importance are critical to prevent the spread of infectious diseases like typhoid and can successfully be accomplished through simple picture pamphlets and school programmes to spread awareness. ${ }^{22}$ The education provided should specifically focus on how typhoid is spread, the prevention of it spread through hand washing and basic hygiene, minimising exposure to contacts, and vaccination for susceptible populations. ${ }^{5}$ In the authors' case, the patient and his family were counselled regarding the implementation of these basic hygienic practices to prevent the transmission of typhoid. 
Vaccination against typhoid exists in two forms: an injectable vaccine for those over 2 years of age and a live attenuated oral vaccine for those over 5 years of age; a third new conjugate type vaccine was prequalified by the WHO in 2017 for use in children as young as 6 months. ${ }^{23}$ The NIH Islamabad has listed the conditions in which the vaccine is justified including for those in direct contact with patients and carriers, healthcare workers who are exposed due to their profession, "institutions where the appropriate hygienic condition cannot be met, areas of high endemic incidence," and travellers. ${ }^{24}$ The combination of these efforts can become a powerful tool to raise awareness, prevent disease, and promote healthy living styles.

\section{CONCLUSION}

In conclusion, as the number of XDR typhoid cases rise it is becoming increasingly necessary to take action under the guidance of the $\mathrm{NIH}$ Islamabad and $\mathrm{WHO}$ at a local level in order to effectively minimise the spread of drug resistant communicable diseases. The power of community education programmes and locally set-up campaigns for the promotion of public health should not be underestimated. Although the resistant strain has already emerged, healthcare professionals can still help to curb its rapid spread, even the communities with the least resources, by emphasising and promoting the implementation of simple preventive measures.

This case highlighted the importance of high clinical suspicion and prompt diagnostic investigations including a blood culture. Compared to other existing literature that address special populations, such as pregnant females or cases in which patients present with pathognomonic features such as rose spots, the authors' case presented with no clear indication typhoid and care was taken to follow the proper guidelines regarding thorough investigations. ${ }^{6,25}$ There were also many studies revealed during the authors' literature search that emphasised outbreaks and the global burden of typhoid cases; however, by providing this perspective of an average young male, the authors are aiming to add a case study into the existing literature to increase insight at the individual level specifically from a geographical area like Pakistan, where extensively drug resistant typhoid took its origin. 18,19,21 Lastly, the authors believe that, through this study, healthcare providers will be reminded to use antibiotics appropriately and only when necessary, to impede further antibiotic resistance.

\section{References}

1. Akram J et al. Extensively drugresistant (XDR) Typhoid: evolution, prevention, and its management. Biomed Res Int. 2020;2020:6432580.

2. Akullian A et al. Environmental transmission of Typhoid Fever in an urban slum. PLoS Negl Trop Dis. 2015;9(12):2-5.

3. Johnson $\mathrm{R}$ et al. Typhoidal Salmonella: distinctive virulence factors and pathogenesis. Cellular Microbiology. 2018;20(9):1-14.

4. Yang YA. et al. Why is eradicating typhoid fever so challenging: implications for vaccine and therapeutic design. Vaccines. 2018;6(3):1-2.

5. World Health Organization. Typhoid fever - Islamic Republic of Pakistan. 2018. Available at: https://www.who. int/csr/don/27-december-2018typhoid-pakistan/en/ Date accessed: 28th October 2019.

6. Azhar $A B$ et al. The implications of extensive drug-resistant typhoid fever: a case report. Cureus.
2019;11(6):1-6.

7. Dall C. Study: XDR typhoid in Pakistan carries added resistance genes. CIDRAP. University of Minnesota. 2018. Available at: https://www.cidrap.umn.edu/newsperspective/2018/02/study-xdrtyphoid-pakistan-carries-addedresistance-genes Date accessed: 28 October 2019.

8. Larik EA et al. Weekly field epidemiology report NIH Islamabad. 2019. Available at: https:// www.nih.org.pk/wp-content/ uploads/2019/09/36-FELTPPakistan-Weekly-EpidemiologicalReport-Sept-02-08-2019-.pdf Date accessed: 28 October 2019.

9. Syed A et al. Weekly field epidemiology report $\mathrm{NIH}$ Islamabad. 2019. Available at: https:// www.nih.org.pk/wp-content/ uploads/2019/10/40-FELTP-PakistanWeekly-Epidemiological-ReportSept-30-Oct-06-2019.pdf Date accessed: 28 October 2019.
10. Medical Microbiology \& Infectious Diseases Society of Pakistan. Typhoid Management Guidelines - 2019. Available at: https://www.mmidsp.com/ typhoid-management-guidelines-2019/ Date accessed: 14 July 2021.

11. Klemm EJ et al. Emergence of an extensively drug-resistant Salmonella enterica serovar Typhi clone harboring a promiscuous plasmid encoding resistance to fluoroquinolones and thirdgeneration cephalosporins. mBio. 2018;9(1):1-10.

12. Bhutta ZA et al. World Health Organization. Antimicrobial resistance in Typhoid: implications for policy \& immunization strategies. Center for Global Child Health. 2017. Available at: https://www.who.int/ immunization/sage/meetings/2017/ october/3_Bhutta_Typhoid_ SAGE_16Oct2017.pdf?ua=1 Date accessed: 28 October 2019.

13. Memon A et al. Frequency and antimicrobial resistance pattern of 
extensive-drug resistance Salmonella Typhi isolates. JMID. 2020;10(2):68-74.

14. Center for Infectious Disease Research and Policy. CDC issues health advisory on extensively drug-resistant typhoid. Available at: https://www.cidrap.umn. edu/news-perspective/2021/02/cdcissues-health-advisory-extensivelydrug-resistant-typhoid Date accessed: 14 July 2021.

15. Dyson ZA et al. Antibiotic resistance and typhoid. Clin Infect Dis. 2019;68(2):165-70.

16. National Institute of Health, Islamabad. Advisory for prevention and treatment of Typhoid Fever. 2018. Available at: https://www.nih.org. pk/wp-content/uploads/2018/08/ Advisory-for-prevention-andtreatmentof-Typhoid-Fever-10August-2018.pdf Date accessed: 28 October 2019
17. National Institute of Health Islamabad. Advisory for prevention and treatment of XDR Typhoid. 2018. Available at: https://www.nih.org. $\mathrm{pk} / \mathrm{wp}$-content/uploads/2019/02/ Advisory-for-Typhoid-5-oct.pdf. Date accessed: 29 October 2019.

18. Qamar FN et al. Outbreak investigation of ceftriaxone-resistant Salmonella enterica serotype Typhi and its risk factors among the general population in Hyderabad, Pakistan: a matched case-control study. Lancet Infect Dis. 2018;18(12):1368-76.

19. Siddiqui TR et al. High prevalence of typhoidal Salmonella enterica serovars excreting food handlers in Karachi-Pakistan: a probable factor for regional typhoid endemicity. J Health Popul Nutr. 2015;33(1):1-9.

20. Pollard AJ et al. The time is now to control typhoid. Clin Infect Dis.
2019:68(Suppl 2):S47-9.

21. Crump JA et al. The global burden of typhoid fever. Bull World Health Organ. 2004;82(5):346-53.

22. Rasheed MK et al. Extensively drugresistant typhoid fever in Pakistan. The Lancet Infectious Diseases. 2019;19(3):242-3.

23. World Health Organization. Typhoid 2018. Available at: https://www.who.int/ news-room/fact-sheets/detail/typhoid Date accessed: 5 January 2020.

24. National Institute of Health Islamabad. Typhoid vaccine (TAB). Available at: https://www.nih.org.pk/productscustom/typhoid-vaccine-tab/ Date accessed: 28 October 2019

25. Petrin CE et al. Drug-resistant salmonella typhi in Pakistan. Clin Pediatr (Phila). 2020;59(1):31-3. 